\title{
Introduction of Extra Copy of Oxytetracycline Resistance Gene otrB Enhances the Biosynthesis of Oxytetracycline in Streptomyces rimosus
}

Xiaohe Chu' ${ }^{1,2}$, Zijing Zhen ${ }^{1}$, Zhenyu Tang ${ }^{1}$, Yingping Zhuang ${ }^{1}$, Ju Chu ${ }^{1}$, Siliang Zhang ${ }^{1}$ and Meijin Guo $^{1 *}$

${ }^{1}$ State Key Laboratory of Bioreactor Engineering, East China University of Science and Technology, Shanghai 200237, P.R. China

${ }^{2}$ Zhejiang Shenghua Biok Biology Co., Zhejiang 313220, P.R. China

\begin{abstract}
The aromatic polyketide antibiotic oxytetracycline (OTC) is produced by Streptomyces rimosus as an important secondary metabolite. Enhancement of self-resistance is one effective way to improve antibiotic production in Streptomyces spp. In the present study, we aimed to improve the production of OTC by introducing extra copies of the OTC resistance genes, otrA and otrB, into the chromosome of the industrial strain of S. rimosus (SRI). First, otrA and otrB were cloned and ligated with pSET152 to generate the recombinant plasmids PSET152-otrA/otrB, the demethylated pSET152-otrA/otrB by Escherichia coli ET12567(pUZ8002) were then introduced into SRI to yield otrA/ otrB knock-in mutants: SRI-A (otrA) and SRI-B (otrB). Ten selected mutants and the parent SRI strain were cultured in shake flasks. Production of OTC was increased by $67 \%$ in one SRI-B mutant compared with the parent strain, suggesting that the enhancement of resistance gene otrB in the antibiotic producer is an effective way to improve OTC biosynthesis. However, introduction of extra copy of otrB could retard growth of mutant cells.
\end{abstract}

Keywords: Antibiotic; Aromatic polyketide; Oxytetracycline (OTC) Biosynthesis; Self-Resistance

\section{Introduction}

Streptomyces spp. are filamentous Gram-positive aerobic soildwelling bacteria that belong to the family Streptomycetaceae and the order Actinomycetales. Streptomyces spp. and closely related genera have the ability to coordinate the production of a wide variety of secondary metabolites during morphological development [1]. Many of these secondary metabolites have antibiotic properties. Current methods employed to increase the antibiotic productivity of industrial microorganisms range from classical random mutagenesis studies performed in conjunction with the optimization of large-scale industrial fermentations. For more rational approaches, metabolic engineering is a common method used by researchers to regulate the production of many antibiotics. For example, genetic modifications of primary metabolic fluxes can lead to increases in the productivity of antibiotic synthesis $[2,3]$, since the availability of biosynthetic precursors is a key factor that determines their production. To date, many studies have reported the improvement of antibiotic production by engineering the availability of certain precursors in the producer organisms [4-6]. Other methods, such as the optimization of fermentation conditions, are also available to regulate antibiotic production [7].

The aromatic polyketide antibiotic oxytetracycline (OTC) is produced by Streptomyces rimosus as an important secondary metabolite. There are three resistance genes in the OTC biosynthesis cluster,

\begin{tabular}{|l|l|l|}
\hline Strains or plasmids & Function & Source \\
\hline E. coli Top10 & plasmid amplification & our laboratory \\
\hline E. coli ET12567(pUZ8002) & plasmid demethylation & John Innes Center, UK \\
\hline Industrial S. rimosus (SRI) & OTC producer & $\begin{array}{l}\text { Shanxi Datong Antibiotic } \\
\text { Company }\end{array}$ \\
\hline $\begin{array}{l}\text { pMD19T } \\
\text { pMD19T-otrA } \\
\text { pMD19T-otrB }\end{array}$ & $\begin{array}{l}\text { gene amplification } \\
\text { gene amplification } \\
\text { gene amplification }\end{array}$ & $\begin{array}{l}\text { TARAKA, Japan } \\
\text { this study } \\
\text { this study }\end{array}$ \\
\hline pSET152 & gene integration & \\
\hline $\begin{array}{l}\text { pSET152-otrA } \\
\text { pSET152-otrB }\end{array}$ & $\begin{array}{l}\text { gene integration } \\
\text { gene integration }\end{array}$ & $\begin{array}{l}\text { this study } \\
\text { this study }\end{array}$ \\
\hline
\end{tabular}

Table 1: Strains and plasmids used in this present study. namely $\operatorname{otr} A, \operatorname{otr} B$ and $\operatorname{tr} C$, of which $\operatorname{otr} A$ and $\operatorname{tr} B$ are located at either end of this cluster. otrA changes the conformation of the $30 \mathrm{~S}$ ribosome non-covalently and prevents the binding of OTC . Furthermore, otrA may be a substitute for the regulatory elongation factor [8], and otrB encodes a membrane transport protein that aids the transportation of OTC out of the cell. The otrB sequence shares great similarity with other transport genes, including tetA from Tn10 [9]. However, the function of $\operatorname{tr} C$ remains to be elucidated. A traditional mutation program has resulted in the improvement of OTC production from $2 \mathrm{~g}^{-1}{ }^{-1}$ to 80 g. $\mathbf{l}^{-1}$, and OTC production can also be improved by disrupting the zwf (coding glucose-6-phosphate dehydrogenase) gene [10]. Nevertheless, there is no information concerning the effects of $\operatorname{tr} A, \operatorname{otr} B$ and $\operatorname{otr} C$ on OTC production.

In this present study, we aimed to investigate the influence of otrA and $\operatorname{tr} B$ on OTC production by introducing extra copies of these resistance genes into the genome of the industrial strain of $S$. rimosus $(S R I)$. Herein, it is shown that production of OTC was increased in the otrB mutant, though an extra copy of otrA had no effect on OTC production.

\section{Materials and Methods}

\section{Bacterial strains, media, and plasmids}

The strains and plasmids used in this present study are listed in

*Corresponding author: Meijin Guo, State Key Laboratory of Bioreactor Engineering, P.O. box 329, East China University of Science and Technology, 130 Meilong Rd., Shanghai 200237, P.R. China; Tel: +86 21 64251131; Fax: +86 21 64253702, Email: guo_mj@ecust.edu.cn

Received February 02, 2012; Accepted February 27, 2012; Published March 01,2012

Citation: Chu X, Zhen Z, Tang Z, Zhuang Y, Chu J, et al.(2012) Introduction of Extra Copy of Oxytetracycline Resistance Gene otrB Enhances the Biosynthesis of Oxytetracycline in Streptomyces rimosus. J Bioprocess Biotechniq 2:117 doi: 10.4172/2155-9821.1000117

Copyright: (C) 2012 Chu X, et al. This is an open-access article distributed unde the terms of the Creative Commons Attribution License, which permits unrestricted use, distribution, and reproduction in any medium, provided the original author and source are credited. 
Citation: Chu X, Zhen Z, Tang Z, Zhuang Y, Chu J, et al.(2012) Introduction of Extra Copy of Oxytetracycline Resistance Gene otrB Enhances the Biosynthesis of Oxytetracycline in Streptomyces rimosus. J Bioprocess Biotechniq 2:117 doi: 10.4172/2155-9821.1000117

Page 2 of 4

\begin{tabular}{|c|c|c|}
\hline Primer & Sequence & $\begin{array}{l}\text { Digestion } \\
\text { site }\end{array}$ \\
\hline $\begin{array}{l}\text { P1 (otrA-F) } \\
\text { P2(otrA- } \\
\text { R) }\end{array}$ & $\begin{array}{l}\text { 5' } \text { CGCCATATGATGAACAAGCTGAATCTGGG 3' } \\
5^{\prime} \text { GGAAGCTTTCTAGATCACACGCGCTTGAGC } 3{ }^{\prime}\end{array}$ & $\begin{array}{l}\text { Ndel } \\
\text { Xbal }\end{array}$ \\
\hline P3(otrB-F) & 5' CGCCATATGGTGTCATCCGCAAATCCG 3' & Ndel \\
\hline $\begin{array}{l}\text { P4(otrB- } \\
\text { R) }\end{array}$ & 5' CCAAGCTTGCTCTAGATCAGGCGTCCGACGC 3' & Xbal \\
\hline P5(attB-F) & 5' GTTCACCAACAGCTGGAGGC 3' & \\
\hline P6(attB-R) & 5' CGTCATGCCCGCAGTGACC 3' & \\
\hline
\end{tabular}

Table 2: Primers used in this present study.

Table 1. Organisms were grown at $37^{\circ} \mathrm{C}$ in Luria-Bertani medium $(1 \%$ tryptone, $1 \% \mathrm{NaCl}, 0.5 \%$ yeast extract), and standard procedures were used for transformations [11]. Escherichia coli transformants were se-

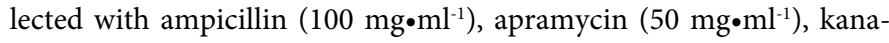
mycin $\left(10 \mathrm{mg} \cdot \mathrm{ml}^{-1}\right)$ or chloramphenicol $\left(25 \mathrm{mg} \mathrm{ml}^{-1}\right)$. SRI (S. rimosus M4018) was grown and manipulated as described previously [12].

\section{Construction of recombinant plasmids and SRI mutants}

Primers: All the primers used are listed in Table 2.

Construction of recombinant plasmids: The otrA $(2.1 \mathrm{~kb})$ and $\operatorname{otrB}(1.7 \mathrm{~kb})$ gene fragments were amplified using primers $\mathrm{P} 1$ and $\mathrm{P} 2$ or P3 and P4. Genomic DNA of SRI was used as the template. The fragments were cloned into the pMD19T vector to yield pMD19T-otrA/ $o t r B$, and then transformed into competent cells of E. coli Top10. Recombinant clones were screened by white-blue plaque selection and recombinant plasmids were analyzed by both single and double restriction enzyme digestion. pMD19T-otrA/otrB were digested with $\mathrm{NdeI}$ and $X b a \mathrm{I}$, and then cloned into pSET152, which was digested with the same restriction enzymes to give pSET152-otrA/otrB. Then, the plasmids were transferred into E. coli ET12567 for demethylation and stored at $-20^{\circ} \mathrm{C}$ until later use.

Gene enhancement and identification of mutants: Demethylated pSET152-otrA/otrB were electroporated into SRI competent cells at $2 \mathrm{kV}, 25 \mu \mathrm{F}$ and $400 \Omega$. Exconjugants were selected on tryptone soy agar plates containing apramycin $500 \mu \mathrm{g} \bullet \mathrm{mL}^{-1}$ and incubated at $30^{\circ} \mathrm{C}$ for 4-6 days. Mutants were confirmed by polymerase chain reactions (PCR) using $\operatorname{aprF}(\mathrm{P} 5)$ and $\operatorname{aprR}(\mathrm{P} 6)$ as primers.

\section{Fermentation experiments}

A spore suspension was inoculated into $30 \mathrm{ml}$ of seed medium containing glucose $\left(10 \mathrm{~g} \bullet \mathrm{l}^{-1}\right)$, yeast extract $\left(0.5 \mathrm{~g}^{\circ} \mathrm{l}^{-1}\right)$, tryptone $\left(15 \mathrm{~g}^{-1} \mathrm{l}^{-1}\right)$, sucrose $\left(2.8 \mathrm{~g}^{-1}{ }^{-1}\right)$ and calcium carbonate $\left(0.1 \mathrm{~g}^{-l^{-1}}\right)$. The first seed cultures were grown for 3 days at $260 \mathrm{rpm}$ and $30^{\circ} \mathrm{C}$. Then, $2 \mathrm{ml}$ of the first seed culture was inoculated into $50 \mathrm{ml}$ of fermentation medium [12] in a $500-\mathrm{ml}$ shaking flask with a spring. The second cultures were grown for 8 days at $260 \mathrm{rpm}$ and $30^{\circ} \mathrm{C}$.

For determination of dry cell weight, $5-\mathrm{ml}$ samples of each culture were collected every $24 \mathrm{~h}$ and dried at $105^{\circ} \mathrm{C}$ to constant weight. OTC production in vivo and in vitro was analyzed by high performance liquid chromatography according to reference 9 .

\section{Results}

\section{Validation of the introduction of extra otrA and otrB genes}

The recombinant plasmids pSET152-otrA and pSET152-otrB were verified by $X b a \mathrm{I}$ and $N d e \mathrm{I}$ digestion (Figure $1 \mathrm{~A}$ ). As indicated in Figure $1 \mathrm{~B}$, the positive clones of otrA and otrB transformants showed strong signals at $750 \mathrm{bp}$ (apramycin resistance gene), while the wild-type pSET152 and pKC1139 showed no bands. After site-specific integration, the entire recombinant plasmid should be inserted into the genome of $S R I$ as shown in Figure 1C. Then, positive clones were identified by cross-over sites (Figure 2) using the primers otr $A-\mathrm{F}$ and $a t t B-\mathrm{R}$ or $a t t B-F$ and $a p r-\mathrm{R}$.

\section{Screening of the high-productivity mutants}

Eight SRI-A mutants (containing an extra copy of otrA) and 12 $S R I-B$ mutants (containing an extra copy of $\operatorname{tr} B$ ) were chosen for the shake flask experiments. After 8 days of fermentation, there were no obvious differences in the levels of OTC in the SRI parent strain, the $S R I-A$ mutants and most of the SRI-B mutants (Figure 3). However,
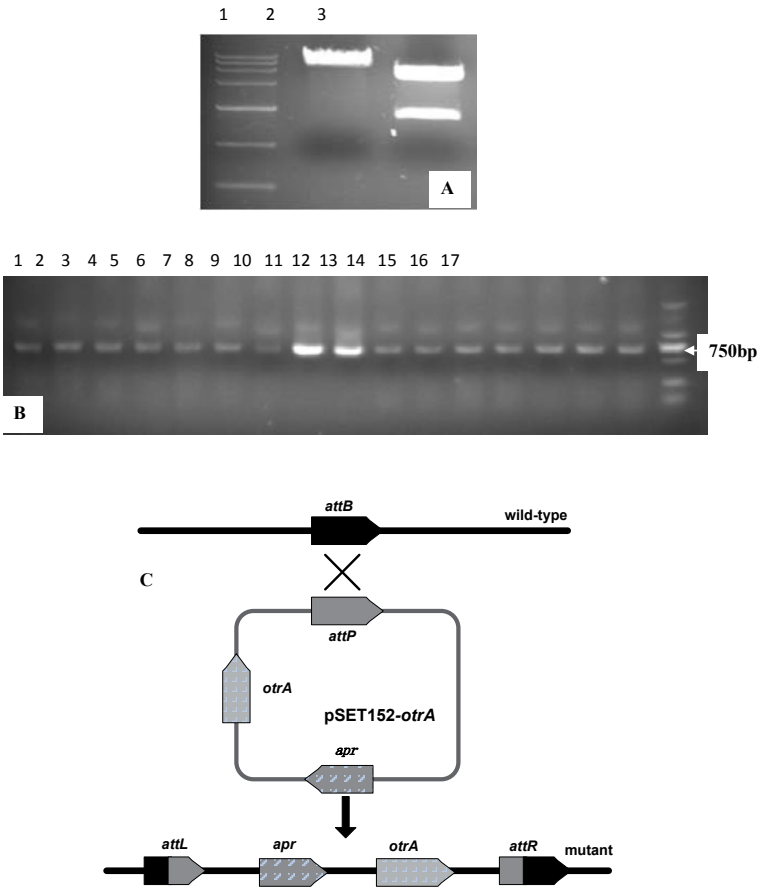

Figure 1: Generation and validation of the introduction of extra otrA and otrB genes.

A.Digestion of pSET152-otrB. Lane 1: marker DL15000; lane 2: pSET152otrB digested with Ndel; lane 3: pSET152-otrB digested with Xbal and Ndel. B. PCR identification of SRI mutants. Lanes 1-7: SRI-A mutants; lane 8-9: positive control(750bp for apramycin resistance gene); lane 10-16: SRI-B mutants; lane 17: marker III.

C. Site-specific integration of otrA in the SRI genome.

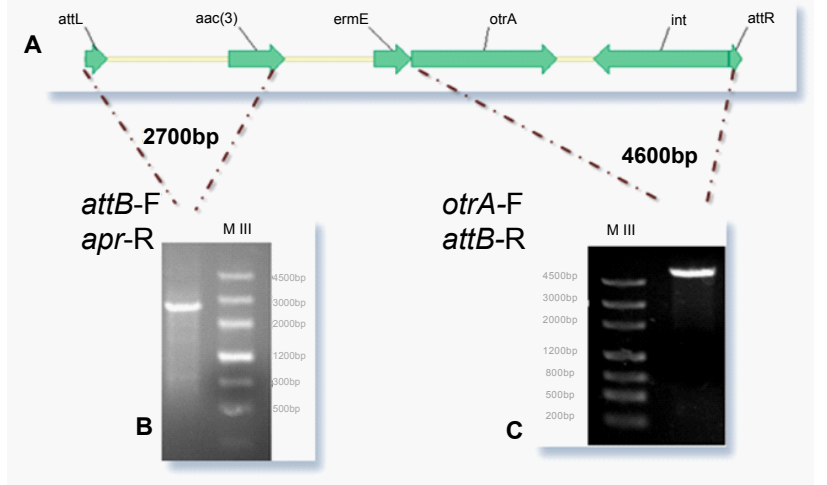

Figure 2: PCR identification of site-specific integration. 
Citation: Chu X, Zhen Z, Tang Z, Zhuang Y, Chu J, et al.(2012) Introduction of Extra Copy of Oxytetracycline Resistance Gene otrB Enhances the Biosynthesis of Oxytetracycline in Streptomyces rimosus. J Bioprocess Biotechniq 2:117 doi: 10.4172/2155-9821.1000117

Page 3 of 4

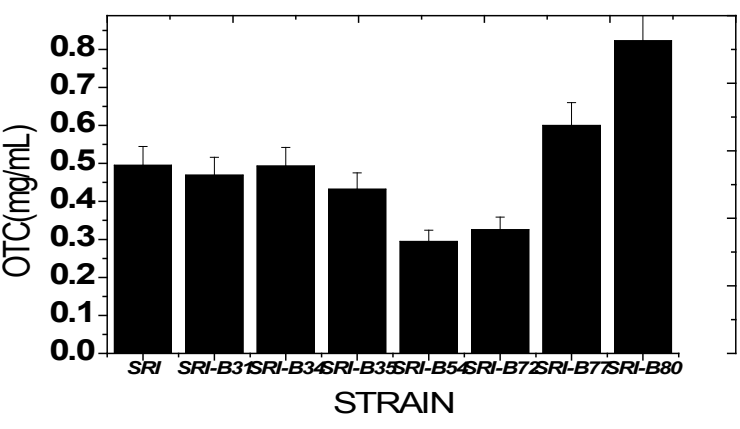

Figure 3: OTC levels in SRI, and SRI-A and SRI-B mutants.

front

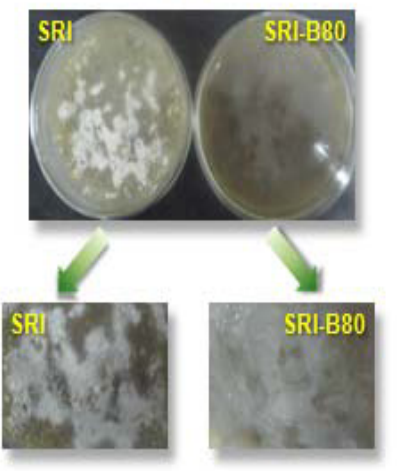

Figure 4: Pigment and spore formation by SRI-B80 and SRI.
OTC production was $67 \%$ higher in strain SRI-B80 compared with the control, indicating that $S R I-B 80$ had high production of OTC.

Knowing that Streptomyces spp. spore formation is important for growth and the production of secondary metabolites [13], spore formation was compared between the SRI and SRI-B80 strains. After 6 days of incubation, mature spores were found on the surface of the SRI colony, but the SRI-B80 spores were malformed and immature. The onset of morphological differentiation generally coincides with the production of secondary metabolites and resistance genes are often co-regulated with these metabolites [14]. Indeed, spore formation is very closely related with the expression of resistance genes. $\operatorname{otr} B$ may be a negative regulator of spore formation and morphological differentiation. Moreover, the level of pigments produced by some Streptomyces spp. can reflect the magnitude of antibiotic production. Visual inspection of agar plates revealed that $S R I-B 80$ produced more pigment than $S R I$, and this coincided with OTC production (Figure 4).

\section{Growth characteristics of SRI and SRI-B80b}

The physiological characteristics of SRI-B80 were further investigated and compared with the SRI parent. The growth curves of SRI and SRI-B80 are given in Figure 5A. The final biomass of SRI-B80 was markedly lower than $S R I$, indicating that the extra copy of $o t r B$ had a negative effect on growth. Furthermore, SRI-B80 had a slower growth rate, even during exponential growth phase. There are two possible reasons for this. First, the extra copy of otrB may increase the physiological burden of the cells. Second, re-distribution of cellular resources may occurred so that more materials could be directed into OTC biosyn- thesis in the SRI-B80 strain because the specific OTC productivity of $S R I-B 80$ was twice that of $S R I$ after 5 days of fermentation (Figure 5B).

\section{OTC efflux mediated by the extra copy of otrB in SRI-B80}

The intracellular and extracellular concentrations of OTC were determined after fermentation for the SRI and SRI-B80 strains. As expected, SRI-B80 showed enhanced efflux of OTC to some extent. After 8 days of fermentation, the introcellular OTC concentration in SRI-B80 was $13.6 \%$ lower than found in SRI (Figure 6A), which may reduce the risk of cell suicide due to high concentrations of OTC. Concomitantly, OTC concentration outside of the cells was $21.2 \%$ greater for SRI-B80 than detected for SRI. This shows that the extra copy of otrB played an important role in transporting OTC out of the cells. The extra otrB gene in SRI-B80 enabled greater transport of OTC out of the cells and this ensured that OTC in the cells was maintained at sufficiently low concentrations to cause any harm (Figure 6B).

\section{Discussion}

In this present study, extra copies of $\operatorname{otr} A$ and $\operatorname{otr} B$ were introduced into the genome of SRI. The extra copy of otrA did not exert any effects on OTC production; however, the extra copy of otrB enhanced OTC production markedly. Nevertheless, the SRI-B80 mutant had a slower growth rate, and spore formation and morphological differentiation were also affected, which suggests that $\operatorname{otr} B$ may be a multi-functional regulator in SRI. Through comparison of OTC concentrations in vivo and in vitro for $S R I$ and $S R I-B 80$, the extra otrB gene was shown to facilitate the intracellular efflux of OTC. Thus, the lower concentrations of OTC in vivo decrease the toxicity of the excess OTC, which may in turn stimulate the cells to produce more of this metabolite.

Many microbes can synthesize potentially toxic secondary metabolites (mainly antibiotics), and so there must exist pathways or mech-
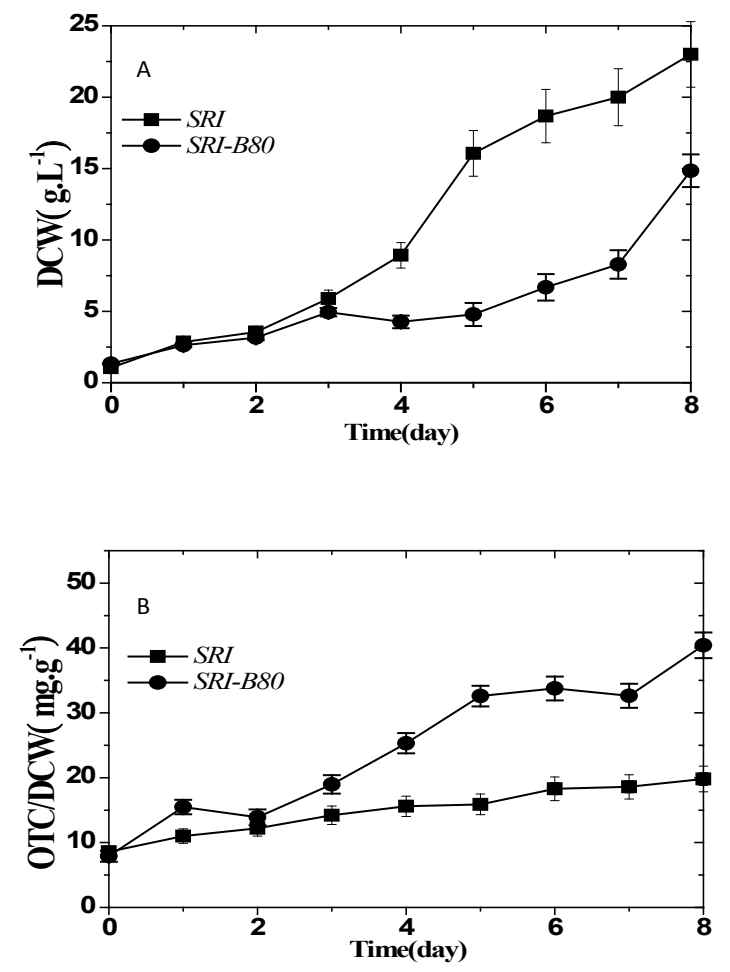

Figure 5: Growth and specific OTC productivities of SRI and SRI-B80. 
Citation: Chu X, Zhen Z, Tang Z, Zhuang Y, Chu J, et al.(2012) Introduction of Extra Copy of Oxytetracycline Resistance Gene otrB Enhances the Biosynthesis of Oxytetracycline in Streptomyces rimosus. J Bioprocess Biotechniq 2:117 doi: 10.4172/2155-9821.1000117

Page 4 of 4
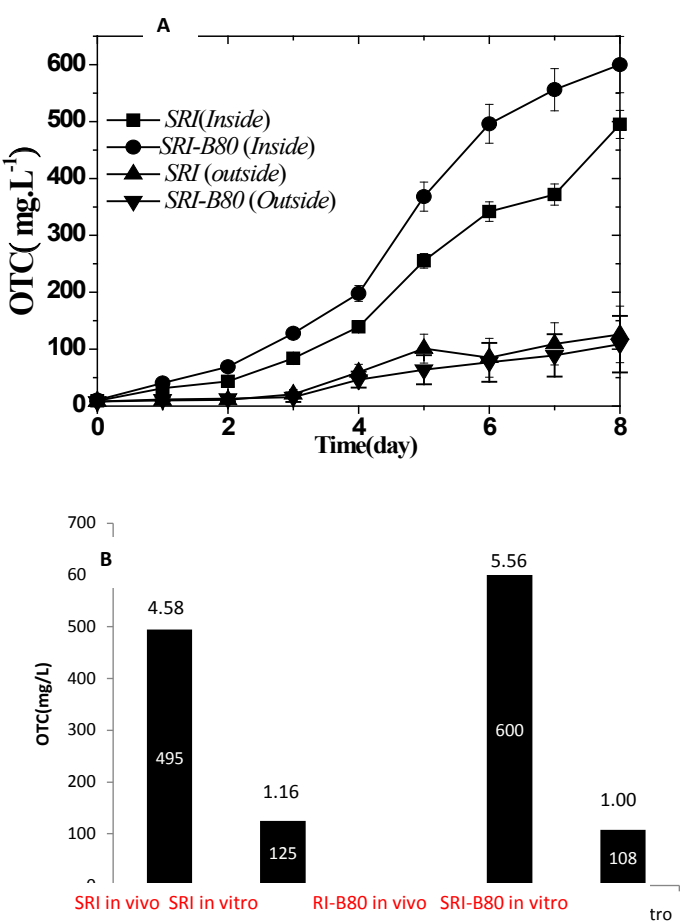

Figure 6: Intracellular and extracellular concentrations of OTC in SRI and SRI-B80.

anisms that protect the producer. Until now, various self-protection mechanisms have been reported [15], and by enhancing these selfprotection mechanisms, the production of many antibiotics has been increased in various microorganisms. For example, Malla et al. [14] overexpressed the resistance genes $d r r A, d r r B$ and $d r r C$ in Streptomyces peucetius ATCC 27952, such that the recombinant strains produced more doxorubicin (DXR) than the parental strain. Indeed, there was a 2.2-fold increase in DXR in the $\operatorname{dr} A B$ mutant, a 5.1-fold increase in the $d r r C$ mutant and a 2.4 -fold increase in the $d r A B C$ mutant. Similarly, Olano et al. [16] demonstrated that improvements in self-resistance could increase the production of bioactive secondary metabolites in the actinomycetes. Thus, the introduction of extra resistance genes is an effective approach to enhance antibiotic production.

Although an extra copy of otrB improved OTC production, the additional copy of otrA had little or no effect on this parameter. This may be because otrA in SRI produces sufficient enzyme to modify the ribosome and provide a safe environment. Therefore, otrA may be saturating, but this assumption requires further investigation. Moreover, the observation that $S R I-B 80$ has a slow rate of sporulation is consistent with the findings of Davies [17] and Den Hengst et al [18]. Specific growth states, such as biofilm formation, anaerobiosis and sporulation, can differentially impact on cell susceptibility to antimicrobials. It can be concluded that a series of genes that regulate $\operatorname{tr} B$ may have altered expression in SRI-B80, and these changes may have knock-on effects on growth and sporulation in these mutants. Since the regulation network in these cells is very complicated, further experiments are needed to understand the relations between all of these affected genes.

\section{Acknowledgements}

This work was supported by grants from the Fundamental Research Funds for the Central Universities (ECUST), the Outstanding Youth Talent Plan of East China
University of Science and Technology (ECUST) and the Open Project Program of the State Key Laboratory of Bioreactor Engineering, ECUST (No.2060204).

\section{References}

1. Thompson CJ, Fink D, Nguyen LD (2002) Principles of microbial alchemy: insights from the Streptomyces coelicolor genome sequence. Genome Biol 3 . 1020

2. Nielsen $\mathrm{J}$ (1998) The role of metabolic engineering in the production of secondary metabolites. Curr Opin Microbiol 1: 330-336.

3. Butler MJ, Bruheim P, Jovetic S, Marinelli F, Postma PW, et al. (2002) Engineering of primary carbon metabolism for improved antibiotic production in Streptomyces lividans. Appl Environ Microbiol 68:4731-4739.

4. Reeves AR, Cernota WH, Brikun IA, Wesley RK, Weber JM (2004) Engineering precursor flow for increased erythromycin production in Aeromicrobium erythreum. Metab Eng 6: 300-312.

5. Wei X, Yunxiang L, Yinghua Z (2006) Enhancement and selective production of oligomycin through inactivation of avermectin's starter unit in Streptomyces avermitilis. Biotechnol Lett 28: 911-916.

6. Ryu YG, Butler MJ, Chater KF, Lee KJ (2006) Engineering of primary carbohydrate metabolism for increased production of actinorhodin in Streptomyces coelicolor. Appl Environ Microbiol 72: 7132-7139.

7. Avignone Rossa C, White J, Kuiper A, Postma PW, Bibb M, et al. (2002) Carbon flux distribution in antibiotic-producing chemostat cultures of Streptomyces lividans. Metab Eng 4: 138-150.

8. Doyle D, McDowall KJ, Butler MJ, Hunter IS (1991) Characterization of an oxytetracycline resistance gene, otrA of Streptomyces rimosus. Mol Microbiol 5 2923-2933.

9. McMurry LM, Levy SB (1998) Revised sequence of OtrB (tet347) tetracycline efflux protein from Streptomyces rimosus. Antimicrob Agents Chemother 42 3050.

10. Tang Z, Xiao C, Zhuang Y, Chu J, Zhang S, et al. (2011) Improved oxytetracycline production in Streptomyces rimosus M4018 by metabolic engineering of the G6PDH gene in the pentose phosphate pathway. Enzyme Microbial Technol 49: 17-24

11. Sambrook J, Fritsch EF, Maniatis T (1989) Molecular cloning: a laboratory manual. (2ndedn), New York, Cold Spring Harbor Laboratory Press

12. Kieser T, Bibb MJ, Buttner MJ, Chater KF, Hopwood DA(2000) Practical Streptomyces genetics. John Innes Foundation, Norwich, United Kingdom.

13. Gverzdys TA (2011) The development of protocols to engineer and screen Streptomyces in high throughput to test for the activation of cryptic clusters by the heterologous expression of pleiotropic regulators. Open Access Dissertations and Theses. Paper 6090.

14. Malla S, Niraula NP, Liou K, Sohng JK (2010) Self-resistance mechanism in Streptomyces peucetius: Overexpression of $d r r A, d r r B$ and $d r r C$ for doxorubicin enhancement. Microbiol Res 4: 259-267.

15. Cundliffe E, Demain AL (2010) Avoidance of suicide in antibiotic-producing microbes. J Ind Microbiol Biotechnol 37: 643-672.

16. Olano C , Lombó F, Méndez C, Salas JA (2008) Improving production of bioactive secondary metabolites in actinomycetes by metabolic engineering. Metab Eng 10: 281-292.

17. Crameri R, Davies JE (1986) Increased production of aminoglycosides associated with amplified antibiotic resistance genes. J Antibiot 39: 128-135.

18. Den Hengst CD, Tran NT, Bibb MJ, Chandra G, Leskiw BK, et al. (2010) Genes essential for morphological development and antibiotic production in Streptomyces coelicolor are targets of BIdD during vegetative growth. Mol Microbio 78: 361-379. 